\title{
MODELING MAGNETAR OUTBURSTS: FLUX ENHANCEMENTS AND THE CONNECTION WITH SHORT BURSTS AND GLITCHES
}

\author{
J. A. PONS ${ }^{1}$ AND N. REA ${ }^{2}$ \\ ${ }^{1}$ Department de Física Aplicada, Universitat d'Alacant, Ap. Correus 99, E-03080 Alacant, Spain \\ ${ }^{2}$ Institut de Ciències de l'Espai (CSIC-IEEC), Campus UAB, Facultat de Ciències, Torre C5-parell, E-08193 Barcelona, Spain \\ Received 2011 December 22; accepted 2012 March 19; published 2012 April 9
}

\begin{abstract}
The availability of a large amount of observational data recently collected from magnetar outbursts is now calling for a complete theoretical study of outburst characteristics. In this Letter (the first of a series dedicated to modeling magnetar outbursts), we tackle the long-standing open issue of whether or not short bursts and glitches are always connected to long-term radiative outbursts. We show that the recent detection of short bursts and glitches seemingly unconnected to outbursts is only misleading our understanding of these events. We show that, in the framework of the starquake model, neutrino emission processes in the magnetar crust limit the temperature, and therefore the luminosity. This natural limit to the maximum luminosity makes outbursts associated with bright persistent magnetars barely detectable. These events are simply seen as a small luminosity increase over the already bright quiescent state, followed by a fast return to quiescence. In particular, this is the case for 1RXS J1708-4009, 1E 1841-045, SGR 1806-20, and other bright persistent magnetars. On the other hand, a similar event (with the same energetics) in a fainter source will drive a more extreme luminosity variation and longer cooling time, as for sources such as XTE J1810-197, 1E 1547-5408, and SGR 1627-41. We conclude that the non-detection of large radiative outbursts in connection with glitches and bursts from bright persistent magnetars is not surprising per se, nor does it need any revision of the glitches and burst mechanisms as explained by current theoretical models.
\end{abstract}

Key words: stars: magnetars - stars: neutron - X-rays: bursts

\section{INTRODUCTION}

After the discovery of the first large transient event from XTE J1810-197 (Ibrahim et al. 2004), the study of transient long-term activity of soft gamma repeaters (SGRs) and anomalous X-ray pulsars (AXPs) has provided a new tool to study the physics of strongly magnetized neutron stars (see Mereghetti 2008 and Rea \& Esposito 2011 for recent reviews). At present, thanks to wide field monitors such as the Swift Burst Alert Telescope (BAT) and the Fermi Gamma-ray Burst Monitor, we are currently detecting one or two new outbursts per year, both from known or newly discovered sources. The interpretation of the large amount of observational data accumulated leads to (apparently contradictory) conclusions concerning the connection of large, long-term flux variations (outbursts) with the occurrence of short bursts and/or glitches.

Already, from one of the first outbursts discovered, the connection between the occurrence of glitches, short bursts, and the increase in the persistent flux of 1E 2259+586 was clear (Kaspi et al. 2003; Woods et al. 2004; Zhu et al. 2008). Not only were all three phenomena observed at the same time, but this was also in agreement with a theoretical explanation for how bursts and outbursts are generated. They are thought to be caused by large-scale rearrangements of the crustal and/or magnetospheric field, resulting in the fracture of the neutron star crust when magnetic stresses locally exceed the tensile strength of the crust. The starquake is accompanied by the release of elastic and magnetic energy and may result in renewed magnetospheric activity and additional hot spots on the neutron star surface. This is the plausible cause of spectral changes during outbursts, pulse profile variability, and differences in cooling patterns. The energetics and event frequency depend not only on the strength of the dipolar field, but also on the intensity and geometry of the internal field and the age of the source (Pons \& Perna 2011).
However, although many sources were discovered through their outbursts, and later linked to the occurrence of short bursts and glitches, in many other cases bursts and glitches occurred without the detection of a simultaneous outburst (Dib et al. 2008), or with only very subtle flux variations (Rea et al. 2005). This apparently random connection between glitches/bursts and long outbursts prompted further questions related to the theory behind the crustal fractures, and originated ideas related to the possible magnetospheric origin of bursts not connected with large radiative enhancements (Lyutikov 2006). For all these reasons, disentangling the connection between transient outbursts, glitches, and short and large bursts, has been one of the major issues in the magnetar field in the past few years. In this Letter we discuss the circumstances under which the simultaneous detection of bursts, glitches, and a long outburst is expected. At the same time we give an explanation for the apparent lack of connection in some cases.

\section{OBSERVATIONAL GROUND}

In this section we summarize our current knowledge of magnetar flux variations and their connection with bursting and glitching behavior.

\subsection{Glitches and/or Short Bursts with Very Subtle or No Flux Variability}

1E 1841-045, embedded in the bright SNR Kes 73, is one of the most prolific glitchers among magnetars (Dib et al. 2008). It recently showed a few bursts (Gavriil et al. 2011b). However, its flux was never observed to vary significantly despite long-term monitoring programs with most of the current X-ray satellites (Zhu \& Kaspi 2010; Lin et al. 2011).

Other sources where only very subtle flux changes were observed are 1RXS J1708-4009 and 4U 0142+614 (by a factor $<2$ ). The weak flux variability from 1RXS J1708-4009 has 
been linked to its glitching behavior (Rea et al. 2005; Israel et al. 2007b), but no bursts have been observed thus far from this magnetar. On the other hand, 4U $0142+614$ is one of the first discovered magnetars and has been extensively monitored in the past decade. It showed a few X-ray bursts and a glitch, again with only a subtle increase in luminosity (Gonzalez et al. 2010; Gavriil et al. 2011a).

SGR 1806-20 and SGR 1900+14 are the most prolific X-ray bursters among the magnetar class, and both showed a giant flare: the most energetic events ever observed from Galactic compact objects $\left(L \sim 10^{46} \mathrm{erg} \mathrm{s}^{-1}\right)$. Despite their frequent flaring activity, only very slight flux variations have been observed from them. In particular, SGR 1806-20 showed a subtle increase of its burst rate and its X-ray persistent emission during 2003 and throughout 2004, when the luminosity less than doubled with respect to the "historical" level (Mereghetti et al. 2005; Woods et al. 2007). This period of intense activity culminated with a giant flare (Hurley et al. 2005; Palmer et al. 2005). In 1998, following an intense bursting activity, a large flux increase from SGR 1900+14 was reported (Woods et al. 2001) using data from the RXTE All-Sky Monitor (ASM), but Swift did not measure a large flux increase during a similar intense bursting activity epoch in 2006 (Israel et al. 2008). The low positional and timing accuracy of the ASM, the crowded region where this source lies, and the non-detection of such a large flux increase by any other accurate imaging instrument make us inclined to wait for confirmation of such an event. No glitch has been reported for these two sources, although the large timing noise could have hidden their glitching activity in the timing data. On the other hand, large period derivative changes have been measured, which can be the (non-conclusive) evidence of a missed glitch.

\subsection{Glitches and/or Short Bursts Coincident with an Outburst}

Among the well-monitored magnetar long outbursts, we have evidence of glitches and/or bursts in several cases. 1E 2259+586 is the first long transient event discovered, and the prototype of the connection between outbursts, glitches, and bursts (Kaspi et al. 2003; Woods et al. 2004; Zhu et al. 2008). Nevertheless, we now know that its flux variability was among the least extreme cases. 1E 1048.1-5937 showed another episode of transient flux increase (again not extreme though) connected with X-ray bursts and one glitch (Gavriil \& Kaspi 2004; Tiengo et al. 2005; Tam et al. 2008; Dib et al. 2009).

The most extreme transient events, corresponding with a luminosity increase of a factor of $\sim 100$ or more, have always been observed in coincidence with bursting activity, such as XTE J1810-197 (Ibrahim et al. 2004; Bernardini et al. 2011), 1E 1547-5408 (which showed multiple outbursts; Israel et al. 2010; Ng et al. 2010), CXOU J1647-4552 (Muno et al. 2007), SGR 1627-41 (Mereghetti et al. 2006; Esposito et al. 2008), SGR 0501+4516 (Rea et al. 2009), or the newly discovered SGR 0418+5729 (van der Horst et al. 2010; Esposito et al. 2010; Rea et al. 2010), and SGR 1833-0832 (Göğüş et al. 2010; Esposito et al. 2011). Among those extreme transients, glitches were detected only from CXOU J1647-4552 $\left(\Delta v / v>1.5 \times 10^{-5}\right.$; Israel et al. $2007 \mathrm{a}$; but see also Woods et al. 2007).

\section{CHARACTERIZING THE OUTBURST PROPERTIES AND THE RECOVERY TO QUIESCENCE}

Having revised the observational ground, we turn now to discuss theoretical predictions. We use the two-dimensional cooling code designed to study the magneto-thermal evolution of neutron stars (Aguilera et al. 2008; Pons et al. 2009) to model the rise and decay of luminosity during a magnetar outburst. We refer to these two works for technical details and the microphysics input. To generate an initial pre-burst model, we first evolve a standard magnetar $\left(M=1.4 M_{\odot}, R=11.6\right.$ $\left.\mathrm{km}, B_{p}=(0.5-3) \times 10^{14} \mathrm{G}\right)$, keeping the core temperature fixed, until a stationary solution is obtained. By varying the core temperature and the magnetic field we control the surface temperature, and therefore the quiescent luminosity of the initial model. Once we have a starting model, we inject some fixed amount of energy in a fraction of the crust volume on a very short timescale (hours), and follow the evolution of the thermal structure until it returns to the original state, typically after a few years. On such a short timescale, the magnetic field is practically frozen.

We ran several models varying the total energy injected, the angular size, and the depth of the region where the energy is released. The total energy input varies in the $10^{40}-10^{44}$ erg range, spanning the expected observational estimates and the theoretical predictions (Perna \& Pons 2011). Different depths (from a thin layer to the whole crust) and angular sizes (from $0.2 \mathrm{rad}$ to the entire surface) were tried. After analyzing all the models, the most important conclusions are the following (a longer detailed discussion will be reported in a subsequent paper of this series).

1. Dependence on the depth where the energy is injected. We have found that nearly the totality of the energy injected in the inner crust is efficiently radiated in the form of neutrinos, thus having no effect on the surface temperature and the photon luminosity. This has already been noticed in previous one-dimensional studies (Kaminker et al. 2006), who concluded that the heat source should be located at densities below the neutron drip point $\rho<3-4 \times 10^{11} \mathrm{~g} \mathrm{~cm}^{-3}$ (the outer crust), to have an impact on the thermal luminosity. We confirm that this conclusion remains valid in two-dimensional simulations. Hereafter we use the energy injected in the outer crust $\left(E_{\mathrm{oc}}\right)$ as the reference parameter.

2. Dependence on the angular size. We found that angular heat transport in the outer crust is very inefficient because, in the outer layers, the magnetic field is predominantly radial, and electron conduction across magnetic field lines is strongly suppressed. Angular transport in the inner crust is possible for some particular geometries but, since the energy in the inner crust is rapidly lost by neutrino emission, this has no real effect on the photon luminosity. Hence, the size of the hot spot formed by the energy injection remains almost constant, and only toward the end of the evolution, when the neutron star is close to its original state was a small increase in the surface of the spot observed.

3. Dependence on the energy injection rate. We explored the sensitivity of our results to the variation of the time interval in which the energy is released (from a few minutes to one day). The relevant parameter turned out to be again the total energy $E_{\mathrm{oc}}$, quite independently of the rate at which it is injected. The injection rate affects the rise of the luminosity curve only if the energy is released very close to the surface. In any case, the heat wave needs some time to reach the surface, and the luminosity rise is not instantaneous $(1 \mathrm{hr}$ to 1 day), which is probably too fast to be observable. After reaching the maximum, the cooling curve is independent of the injection rate and reflects a different physics (thermal 

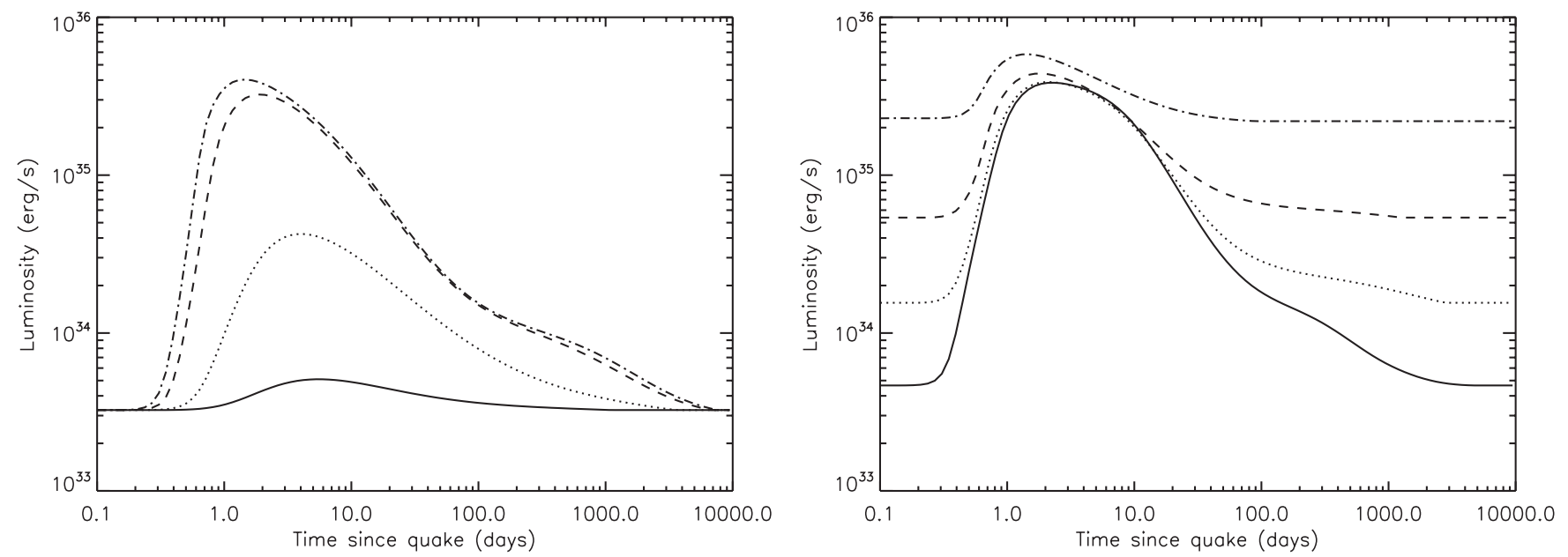

Figure 1. Luminosity vs. time after energy injection. Left panel: effect of the total energy injected. The models correspond to $E_{\mathrm{oc}}=1.7 \times 10^{41} \mathrm{erg}$ (solid line), $1.7 \times 10^{42} \mathrm{erg}$ (dotted line), $1.7 \times 10^{43} \mathrm{erg}$ (dashed line), and $1.7 \times 10^{44} \mathrm{erg}$ (dash-dotted line). Right panel: comparison of models with the same energy injection $\left(E_{\mathrm{oc}}=1.7 \times 10^{44} \mathrm{erg}\right)$ but varying the initial state (quiescent luminosity).

relaxation of the crust). This happens on a longer timescale (months to years).

4. Dependence on the total energy input. A minimum value of $E_{\mathrm{oc}}$ is needed to have a visible effect. For $E_{\mathrm{oc}}<10^{40} \mathrm{erg}$ the event is barely observable as a slight luminosity variation. The most relevant result is an interesting saturation effect for $E_{\mathrm{oc}}>10^{43} \mathrm{erg}$. A larger energy release does not vary the final result. The reason for this saturation is that, as soon as the crust reaches (3-4) $\times 10^{9} \mathrm{~K}$, neutrino processes in the outer crust are strongly reactivated, and the temperature cannot be further increased because the system self-regulates by neutrino emission. However, it should be noted that the two most important neutrino emission processes in this regime are plasmon and pair annihilation (see Yakovlev et al. 2001 and Yakovlev \& Pethick 2004 for reviews on neutrino processes and neutron star cooling), but these two processes in the presence of very strong magnetic fields have not been properly calculated. Further work in this line is needed to fully understand magnetar cooling curves.

Figure 1 (left panel) shows the temporal variation of the luminosity for four representative cases, varying $E_{\mathrm{oc}}$ from $10^{41}$ to $10^{44} \mathrm{erg}$. In all cases heat is deposited in a region with a depth of about $200 \mathrm{~m}$ (between densities $\rho \sim 10^{8}-10^{11} \mathrm{~g} \mathrm{~cm}^{-3}$, and covering a small area $3 \%$ of the star surface, which corresponds to an angle of $0.5 \mathrm{rad}$ ). The delay (a few hours) between the injection of energy and the luminosity peak is caused by the time needed for the internal heat wave to reach the star surface. The saturation when $E_{\mathrm{oc}}>10^{43} \mathrm{erg}$ is clearly visible. A larger energy release does not change the peak luminosity, which only can be increased by enlarging the area affected.

5. Dependence on the initial state. The other fundamental parameter to understand magnetar outbursts is the initial state. The combination of the quiescent luminosity with the saturation effect mentioned above is crucial to understanding magnetar phenomenology. Increasing the total energy injected does not result in higher surface temperatures, which are limited to $0.5-0.6 \mathrm{keV}$ (maybe a short transient flash of a few minutes can reach slightly higher temperatures). Therefore, the maximum thermal luminosity is also limited. ${ }^{3}$ This means that, if the initial state is a very bright magnetar, the luminosity cannot be increased by more than a factor of a few. On the other hand, if the initial state consists of a dim source, we have room to increase its luminosity in 2-3 orders of magnitude.

This is illustrated in Figure 1 (right panel) where we compare results from different models that only differ in the initial state (luminosity). In order to tune the luminosity of the initial state in the stationary regime, we have varied the core temperature between $2 \times 10^{8}$ and $2 \times 10^{9} \mathrm{~K}$, and the value of the poloidal field between $5 \times 10^{13}$ and $2.5 \times 10^{14} \mathrm{G}$, which fixes the heating rate by magnetic field dissipation in the crust. For simplicity, we assumed that no toroidal field is present. The strength of the internal toroidal field is also related to the luminosity of the initial state, but it does not change our conclusions. All of the models have the same energy input: $E_{\mathrm{oc}}=1.7 \times 10^{44} \mathrm{erg}$ in the same region as before. In the figure we can see that, for low quiescent luminosity $\left(L_{q}=3 \times 10^{33}\right.$ $\mathrm{erg} \mathrm{s}^{-1}$ ), a starquake that releases $\approx 10^{44}$ erg produces an increase in the luminosity of two orders of magnitude in about 1 day and its cooling curve can be followed for several years. Conversely, exactly the same type of event in a very bright magnetar $\left(L_{q}=3 \times 10^{35} \mathrm{erg} \mathrm{s}^{-1}\right)$ is barely seen as a small variation of luminosity in a factor of two and lasting only a few days.

\section{DISCUSSION}

We have discussed how the connection between outbursts, short X-ray bursts, and glitches might appear rather erratic. Theoretically, glitches and short X-ray bursts are believed to be correlated to starquakes induced by the progressive increase of magnetic stresses in the crust. When the local conditions are such that the system cannot stand the tension any longer, crustal fractures occur. They may have associated the ejection of particles and the reorganization of the magnetosphere. At

\footnotetext{
3 Resonant Comptonization in the magnetosphere can be very effective in reshaping the spectrum, but it does not vary the total luminosity, which is fixed by the seed thermal photons from the surface. Only in the very extreme case where most of the electrons are ultrarelativistic can the luminosity be visibly enhanced.
} 


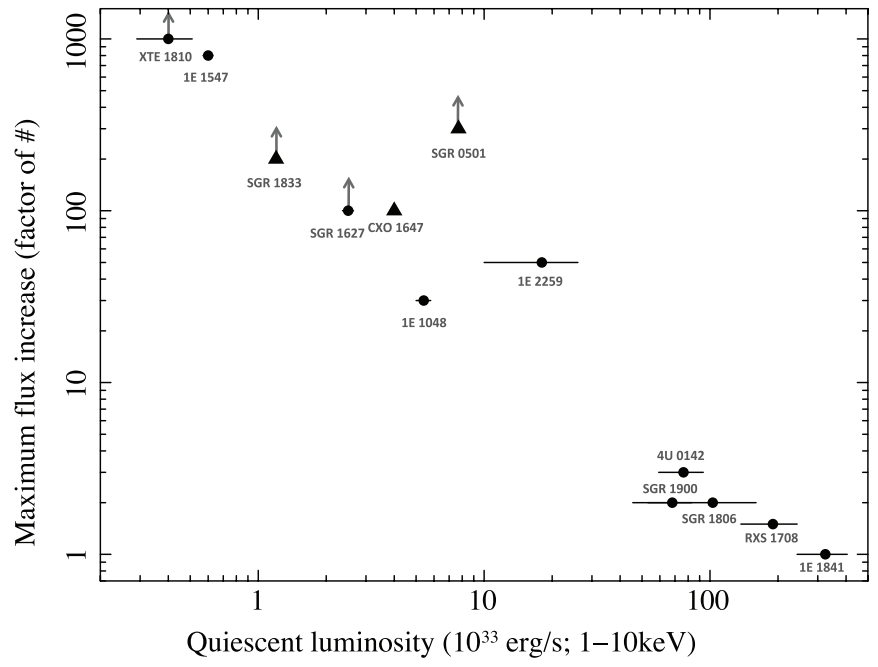

Figure 2. Quiescent luminosity vs. outburst maximum flux increase (all in the 1-10 keV band), for all magnetars showing bursts, glitches, or outbursts. Errors in the measurements include the uncertainties in the flux values and in the distances. Triangles indicate objects with no reported distance uncertainties.

the same time or shortly after, when the heat wave caused by the release of energy reaches the surface, it is also expected to increase the star temperature, and therefore its persistent emission. However, the lack of detection of outbursts correlated with the glitching and bursting activity of several magnetars (see Section 2) posed several questions about the validity of this interpretation, which we now can answer.

In Section 3 we studied the rise of the surface temperature of a magnetar and its subsequent cooling when a certain amount of energy is injected into the outer crust. Because of the strong temperature dependence of the neutrino emission processes, the system can efficiently self-regulate its temperature. The result is an upper limit to the temperature (and luminosity) at the outburst peak: even releasing a much larger amount of energy, the luminosity will reach a maximum between $10^{35}-10^{36} \mathrm{erg} \mathrm{s}^{-1}$, with the precise number depending on the area affected by the event. In other words, any event with an energy release in the outer crust $>10^{43} \mathrm{erg} \mathrm{s}^{-1}$ will show a similar maximum outburst luminosity, regardless of their dipolar magnetic field strength, quiescent luminosity, or any other parameter involved.

In Figure 2 we have plotted the quiescent luminosity of all magnetars that showed glitches and/or bursts, as a function of the maximum persistent flux increase observed in each source. We only consider flux variability on timescales longer than a few days to avoid the contamination from bursts and flares. Furthermore, to select a sample as unbiased as possible, we have neglected flux variations detected with instruments with poor angular and temporal resolution which could not disentangle the contribution from single short bursts (such as RXTE-ASM, and older generation instruments). Among these, we only consider the events for which the outburst decay was also monitored with good resolution instruments (as for XTE J1810-197 and 1E 2259+586).

Although it is almost impossible to have a good quantitative estimate of how many outbursts from magnetars we might have missed in the past years, we note that since the launch of Swift in 2004 (Gehrels et al. 2004), we can rely on a daily coverage of the whole sky with the BAT (which has a field of view of about $1 / 6$ of the sky; $15-150 \mathrm{keV}$ ), and a rapid follow-up with the Swift X-Ray Telescope (0.3-10 keV).
Swift allowed us to collect more than a dozen outbursts in the past eight years, as well as to discover five new magnetars through their outburst activity (see Rea \& Esposito 2011 for a detailed review). This makes us relatively confident of having a good sky coverage and outburst sample, and we believe that only a few events might have been missed during the Swift era.

Looking at Figure 2, a clear trend is present, with brighter objects showing less flux enhancement than dim magnetars. However, we warn that these numbers must be taken with caution due to (1) current distance uncertainties which might well be underestimated, (2) the use of a reduced energy band of, e.g., 1-10 keV, that in combination with the spectral softening during the outburst decay can result in the underestimate of the quiescence luminosity, and (3) the uncertainty in the exact peak flux for many of those objects. For sources having a good pre-outburst monitoring we plot the estimate of the flux enhancement, while we only quote lower limits for the most uncertain cases. In any case, all these caveats may be estimated in about factors of two, and the correlation shown in the figure extends over three orders of magnitude in both axes. Note also that the peak luminosity in all cases is in the expected range of $\sim 10^{35}-10^{36} \mathrm{erg} \mathrm{s}^{-1}$. In particular, fitting the data in Figure 2 (excluding the sources for which we have only lower limits in the peak flux) we find a mean outburst peak luminosity of $\sim 3.5 \times 10^{35} \mathrm{erg} \mathrm{s}^{-1}$.

The general conclusions we can extract from our results can be summarized in the following assertions.

1. The definition of "transient" magnetars (AXPs or SGRs) as opposed to the so-called persistent magnetars is spurious: it only reflects their different quiescent luminosities.

2. Bursts and glitches are probably always accompanied by a radiative enhancement.

3. Given the same typical outburst energetics, large relative flux enhancements can only be observed in faint quiescent objects.

4. Large, long flux enhancements from bright magnetars will never be observed, since their peak radiative luminosities cannot exceed $\sim 10^{36} \mathrm{erg} \mathrm{s}^{-1}$, which in most cases is undetectable. At most, it may simply appear as subtle flux variations (as are the cases of $1 \mathrm{E} 1841-045$ or 1RXS J1708-4009).

The line dividing the historical separation between AXPs and SGRs has been erased during the last decade and now they are thought to represent two regions of the same distribution. With the results presented here, we also show that the same can be said for the separation between "transient" and "persistent" magnetars. As better data are collected and more theoretical work is being done, the separation of magnetars in different classes according to burst activity, timing noise, or spectral properties becomes more and more blurred. This leads to the conclusion that the distribution of neutron stars with relatively high magnetic fields is a continuum with no fundamental intrinsic separation in classes.

This work was partly supported by Compstar, a Research Networking Programme of the European Science Foundation and grants AYA2010-21097-C03-02, GVPROMETEO2009-13 (J.A.P.) and AYA2009-07391, SGR2009-811, iLINK 20110303, and TW2010005 (N.R.). N.R. is supported by a Ramón y Cajal fellowship through Consejo Superior de Investigaciones Científicas (CSIC). 


\section{REFERENCES}

Aguilera, D. N., Pons, J. A., \& Miralles, J. A. 2008, A\&A, 486, 255 Bernardini, F., Perna, R., Gotthelf, E. V., et al. 2011, MNRAS, 418, 638 Dib, R., Kaspi, V. M., \& Gavriil, F. P. 2009, ApJ, 702, 614

Dib, R., Kaspi, V. M., Gavriil, F. P., et al. 2008, ApJ, 673, 1044 Esposito, P., Israel, G. L., Turolla, R., et al. 2010, MNRAS, 405, 1787

Esposito, P., Israel, G. L., Turolla, R., et al. 2011, MNRAS, 416, 205

Esposito, P., Israel, G. L., Zane, S., et al. 2008, MNRAS, 390, L34

Gavriil, F. P., Dib, R., Kaspi, V. M., et al. 2011a, ApJ, 736, 138

Gavriil, F. P., Dib, R., Kaspi, V. M., et al. 2011b, ATel, 3155

Gavriil, F. P., \& Kaspi, V. M. 2004, ApJ, 609, L67

Gehrels, N., Chincarini, G., Giommi, P., et al. 2004, ApJ, 611, 1005

Göğüş, E., Cusumano, G., Levan, A. J., et al. 2010, ApJ, 718, 331

Gonzalez, M. E., Dib, R., Kaspi, V. M., et al. 2010, ApJ, 716, 1345

Hurley, K., Boggs, S. E., Smith, D. M., et al. 2005, Nature, 434, 1098

Ibrahim, A. I., Markwardt, C. B., Swank, J. H., et al. 2004, ApJ, 609, L21

Israel, G. L., Campana, S., Dall'Osso, S., et al. 2007a, ApJ, 664, 448

Israel, G. L., Esposito, P., Rea, N., et al. 2010, MNRAS, 408, 1387

Israel, G. L., Götz, D., Zane, S., et al. 2007b, A\&A, 476, L9

Israel, G. L., Romano, P., Mangano, V., et al. 2008, ApJ, 685, 1114

Kaminker, A. D., Yakovlev, D. G., Potekhin, A. Y., et al. 2006, MNRAS, 371, 477

Kaspi, V. M., Gavriil, F. P., Woods, P. M., et al. 2003, ApJ, 588, L93

Lin, L., Kouveliotou, C., Göğüş, E., et al. 2011, ApJ, 740, L16

Lyutikov, M. 2006, MNRAS, 367, 1594

Mereghetti, S. 2008, A\&AR, 15, 225
Mereghetti, S., Esposito, P., Tiengo, A., et al. 2006, A\&A, 450, 759

Mereghetti, S., Tiengo, A., Esposito, P., et al. 2005, ApJ, 628, 938

Muno, M. P., Gaensler, B. M., Clark, J. S., et al. 2007, MNRAS, 378, L44

Ng, C., Kaspi, V. M., Dib, R., et al. 2010, ApJ, 729, 131

Palmer, D. M., Barthelmy, S., Gehrels, N., et al. 2005, Nature, 434, 1107

Perna, R., \& Pons, J. A. 2011, ApJ, 727, L51

Pons, J. A., Miralles, J. A., \& Geppert, U. 2009, A\&A, 496, 207

Pons, J. A., \& Perna, R. 2011, ApJ, 741, 123

Rea, N., \& Esposito, P. 2011, in Astrophysics and Space Science Proceedings, High-Energy Emission from Pulsars and their Systems, ed. D. F. Torres \& N. Rea (Berlin: Springer), 247

Rea, N., Esposito, P., Turolla, R., et al. 2010, Science, 330, 944

Rea, N., Israel, G. L., Turolla, R., et al. 2009, MNRAS, 396, 241

Rea, N., Oosterbroek, T., Zane, S., et al. 2005, MNRAS, 361, 710

Tam, C. R., Gavriil, F. P., Dib, R., et al. 2008, ApJ, 677, 503

Tiengo, A., Mereghetti, S., Turolla, R., et al. 2005, A\&A, 437, 99

van der Horst, A. J., Connaughton, V., Kouveliotou, C., et al. 2010, ApJ, 711, L1

Woods, P. M., Kaspi, V. M., Thompson, C., et al. 2004, ApJ, 605, 378

Woods, P. M., Kouveliotou, C., Gogus, E., et al. 2001, ApJ, 552, 748

Woods, P. M., Kouveliotou, C., Finger, M. H., et al. 2007, ApJ, 654 , 470

Yakovlev, D. G., Kaminker, A. D., Gnedin, O. Y., \& Haensel, P. 2001, Phys. Rep., 354, 1

Yakovlev, D. G., \& Pethick, C. J. 2004, ARA\&A, 42, 169

Zhu, W., \& Kaspi, V. M. 2010, ApJ, 719, 351

Zhu, W., Kaspi, V. M., Dib, R., et al. 2008, ApJ, 686, 520 